\title{
TRATAMENTO CIRÚRGICO DA ESCOLIOSE IDIOPÁTICA DO ADOLESCENTE UTILIZANDO PARAFUSOS PEDICULARES: ANÁLISE DOS RESULTADOS CLIINICOS E RADIOGRÁFICOS
}

\author{
SURGICAL TREATMENT OF ADOLESCENT IDIOPATHIC SCOLIOSIS USING PEDICLE SCREWS: \\ ANALYSIS OF CLINICAL AND RADIOGRAPHIC RESULTS \\ TRATAMIENTO QUIRÚRGICO DE LA ESCOLIOSIS IDIOPÁTICA DEL ADOLESCENTE UTILIZANDO \\ TORNILLOS PEDICULARES: ANÁLISIS DE LOS RESULTADOS CLIINICOS Y RADIOGRÁFICOS
}

\author{
Alberto Ofenhejm Gotfryd ${ }^{1}$, Fernando José Franzin², Giuliana Rauccl ${ }^{3}$, Nicola Jorge Carneiro Neto ${ }^{3}$, Patrícia Rios Poletto ${ }^{4}$
}

\begin{abstract}
RESUMO
Objetivo: Realizar avaliação clínica e radiográfica de pacientes com escoliose idiopática do adolescente (EIA) submetidos à artrodese por via posterior utilizando parafusos pediculares e correlacionar os resultados por imagem com os escores obtidos através do questionário SRS-24. Métodos: Foram avaliados 25 pacientes com diagnóstico de escoliose idiopática do adolescente, submetidos à artrodese da coluna por via posterior com parafusos pediculares. O seguimento médio foi de 23,7 meses, variando entre 12 e 35 . Analisamos critérios radiográficos referentes à correção das deformidades e aplicamos o questionário SRS-24, específico para esta doença, padronizado pela Scoliosis Research Society. Resultados: A amostra foi composta por 92\% de pacientes do gênero feminino e a média de idade foi de 14,2 anos. A média do índice de Cobb na radiografia ântero-posterior pré-operatória foi de $24,4^{\circ}$ para curva torácica proximal, 54,9 para torácica principal e 38,5 para curva lombar. Os valores angulares médios no pós-operatório foram 11,0; $16,7^{\circ}$ e $12,2^{\circ}$ respectivamente, com porcentagem de correção média da curva torácica principal de 69,9\%. O valor médio final do questionário SRS-24 foi 98,1 pontos. Dois pacientes apresentaram complicações pós-operatórias, entretanto, nenhuma do tipo neurológica. Conclusão: A técnica se mostrou eficaz para tratamento cirúrgico da ElA, proporcionando alto percentual de correção das deformidades, com baixo risco de complicações e resultado clínico satisfatório. Não foi encontrada relação estatisticamente significante entre a porcentagem de correção da curva (torácica principal ou lombar) ou seu valor angular pós-operatório com o valor final do questionário SRS-24 nem com os domínios "satisfação" e "auto-imagem pós-operatória".
\end{abstract}

Descritores: Escoliose; Cirurgia; Adolescente; Resultado de tratamento; Parafusos ósseos.

\begin{abstract}
Objective: To evaluate clinically and radiographically patients with adolescent idiopathic scoliosis (AIS) submitted to posterior arthrodesis using pedicle screws-only constructs and correlate image results with SRS-24 scores. Methods: Twenty five patients with AIS submitted to posterior arthrodesis were evaluated. The follow-up was 23.7 months ranging from 12 to 35. Radiographic criteria were reviewed regarding the deformity correction and a specific questionnaire developed by Scoliosis Research Society (SRS-24) was applied. Results: The sample was composed by $92 \%$ females and $8 \%$ males. The average age was 14.2 years. The Cobb angle average measured by anterior-posterior roentnogegram was $24.4^{\circ}$ at proximal thoracic curve, $54.9^{\circ}$ at principal thoracic and $38.5^{\circ}$ at the lumbar curve. The mean angular values obtained after the surgery were $11.0^{\circ} ; 16.7^{\circ}$ and $12.2^{\circ}$ respectively and the average correction of the principal thoracic curve was $69.9 \%$. The mean SRS-24 scores were 98.1 points. Two patients had post-operative complications; however, there were no neurological complications. Conclusions: The technique showed to be effective on surgical treatment of AIS, with high percentage of deformity correction, low risk of complications and satisfactory clinical results. There was no correlation between the percentage of correction (thoracic or lumbar) and/or the post-operative value of angular deformity and the SRS-24 results, neither with "satisfaction" or "self-image after surgery" domains.
\end{abstract}

Keywords: Scoliosis; Surgery; Adolescent; Treatment outcome; Bone screws.

\section{RESUMEN}

Objetivo: Evaluar clínica y radiográficamente a pacientes portadores de escoliosis idiopática del adolescente sometidos a artrodesis por vía posterior, utilizando solamente tornillos pediculares, y correlacionar el resultado radiográfico con los valores obtenidos a través del cuestionario SRS-24. Métodos: Fueron evaluados 25 pacientes portadores de escoliosis idiopáticas del adolescente, sometidos a artrodesis de la columna por vía posterior. El seguimiento promedio fue de 23,7 meses, con variación entre 12 y 35 . Analizamos criterios radiográficos referentes a la corrección de las deformidades y aplicamos el cuestionario SRS-24, específico para la patología en cuestión, estandarizada por la Scoliosis Research Society. Resultados: La muestra fue compuesta por 92\% de pacientes del sexo

1. Mestre. Médico Assistente do Grupo de Afecções da Coluna Vertebral da Santa Casa da Misericórdia de Santos - Santos, SP. Brasil.

2. Estagiário do Grupo de Afecções da Coluna Vertebral do Departamento de Ortopedia e Traumatologia da Santa Casa da Misericórdia de Santos - Santos, SP. Brasil.

3. Residente do Terceiro Ano do Departamento de Ortopedia eTraumatologia da Santa Casa da Misericórdia de Santos - Santos, SP. Brasil.

4. Doutor em Fisioterapia. Universidade Federal de São Paulo - São Paulo, SP. Brasil.

Trabalho realizado pelo Grupo de Afecções da Coluna Vertebral da Santa Casa da Misericórdia de Santos, SP. Brasil.

Correspondência: Av. Dr. Cláudio Luís da Costa, 50. CEP 11075-900. Santos - SP. Brasil. E-mail : albertocoluna@yahoo.com.br 
femenino y el promedio de edad fue 14,2 años. El promedio del índice de Cobb en la radiografía anteroposterior preoperatoria fue de 24,4 grados para la curva torácica proximal, 54,9 grados para torácica principal y 38,5 grados para la curva lumbar. Los valores angulares promedio en el postoperatorio fueron 11 grados, 16,7 grados y 12,2 grados respectivamente, con un porcentaje de corrección promedio de la curva torácica principal de 69,9\%. El valor promedio final del cuestionario SRS-24 fue de 98,1 puntos. Dos de los pacientes presentaron complicaciones en el postoperatorio, sin embargo ninguna del tipo neurológico. Conclusión: La técnica se mostró eficaz para el tratamiento quirúrgico de la ElA, proporcionando un alto porcentual de corrección de las deformidades, con un bajo riesgo de complicaciones y resultado clínico satisfactorio. No hubo asociación estadísticamente significativa entre el porcentaje de corrección de la curva (torácica o lumbar principal) o su valor postoperatorio para el valor final del cuestionario SRS-24 ni con los dominios "satisfacción" y "autoimagen postoperatoria".

Descriptores: Escoliosis; Cirugía; Adolescente; Resultado del tratamiento; Tornillos óseos.

\section{INTRODUÇÃO}

A escoliose idiopática do adolescente (EIA) é uma deformidade tridimensional da coluna vertebral, com desvios nos planos coronal, sagital e axial. O tratamento desta doença depende da magnitude e da localização da curva, assim como do grau de maturação esquelética do paciente ${ }^{1,2}$. Curvas com angulação maior que 45 graus, medidas pelo método de $\mathrm{Cobb}^{3}$, têm indicação de tratamento cirúrgico nos pacientes com potencial de crescimento. Dentre os objetivos do tratamento estão à redução do valor angular da curva e o equilíbrio do tronco nos planos coronal e sagital, evitando a progressão da deformidade e possíveis complicações cardiopulmonares, além da melhora cosmética.

Os implantes ortopédicos utilizados para a correção das deformidades da coluna vertebral tiveram grande avanço nos últimos 50 anos. Desde a técnica descrita por Harrington ${ }^{4}$ na década de 60, muito se evoluiu sobre o conhecimento da doença e seu tratamento. Em 1984, Cotrel e Dubousset ${ }^{5}$ propuseram um novo tipo de instrumentação segmentar, que permitia a correção da curva nos três eixos anatômicos. A montagem era composta por ganchos e duas hastes paralelas. Alguns anos após, o método recebeu o incremento de parafusos pediculares (PP) lombares, constituindo montagens híbridas. A popularização dos PP ocorreu durante a década de 90, assim como a progressiva utilização de parafusos nos níveis torácicos. Em 1995, Suk et al. ${ }^{6}$ publicaram a primeira série de casos de pacientes tratados só com parafusos pediculares e outros autores reproduziram a técnica, nos anos seguintes, com bons resultados. Atualmente existem evidências disponíveis na literatura comprovando a superioridade das montagens que usam apenas parafusos pediculares, quando comparadas com montagens que utilizam apenas ganchos ou às instrumentações híbridas, em especial com relação ao poder de correção $0^{7-11}$.

A avaliação dos resultados pós-operatórios, tradicionalmente, é feita com base nos parâmetros radiográficos. Para auxiliar, de maneira objetiva, a avaliação clínica pós-operatória, Haher et al. ${ }^{12}$ desenvolveram o Scoliosis Research Society Outcome Instrument (SRS-24). Trata-se de um questionário composto por 24 questões objetivas, divididas em sete tópicos, com a finalidade de abranger diferentes domínios referentes à avaliação pós-operatória. São eles: dor, auto-imagem geral, auto-imagem pós-operatória, função geral, função pós-operatória, nível de atividade e satisfação. Por não se tratar de um questionário validado para língua portuguesa, os autores realizaram a tradução independente do texto original em inglês.

O objetivo deste estudo é avaliar clínica radiograficamente os pacientes portadores de escoliose idiopática do adolescente submetidos a artrodese por via posterior utilizando apenas parafusos pediculares, além de correlacionar o resultado radiográfico com os escores obtidos através do questionário SRS-24.

\section{MATERIAIS E MÉTODOS}

Avaliação prospectiva de 25 pacientes portadores de EIA, submetidos a artrodese vertebral por via posterior, entre maio de 2007 e maio de 2009. Todos os casos foram provenientes do ambulatório de Coluna Vertebral do Serviço de Ortopedia e Traumatologia da Santa Casa da Misericórdia de Santos (SCMS) e operados nesta Instituição pelo mesmo cirurgião. Foram incluídos pacientes com diagnóstico de EIA, de ambos os gêneros, com idade entre 11 e 17 anos e que apresentavam escoliose superior a 45 graus, medida pelo Método de Cobb3 . Excluímos pacientes que apresentavam escoliose secundária a qualquer natureza, pacientes operados com outras técnicas que não fosse o uso exclusivo de parafusos pediculares, reoperações e aqueles que não retornaram para as avaliações ambulatoriais estipuladas. Todos os pacientes foram submetidos à cirurgia por via posterior isolada, com utilização de enxerto ósseo autólogo proveniente dos elementos ósseos vertebrais posteriores e fragmentos de osso esponjoso da crista ilíaca direita. Não foram realizadas toracoplastias.

Os parafusos pediculares foram colocados pela técnica a "mão-livre"13, baseada em marcas anatômicas. O trajeto do parafuso foi palpado com probe, para confirmação do túnel ósseo. Na vigência de violação de alguma parede cortical, o procedimento foi reiniciado e realizado um novo trajeto ósseo. Após a passagem dos parafusos pediculares, obtivemos imagem fluoroscópica para confirmação do correto posicionamento dos mesmos. Apenas parafusos pediculares monoaxiais (de cabeça fixa) foram utilizados nas montagens (Figura 1).

A manobra de correção variou de acordo com o padrão da curva. Optou-se pela "derrotação" da haste da concavidade em pacientes portadores de deformidades tipo Lenke 1 e 3 (King 1, 2 e 3). Para as demais curvas (casos 3 e 7), optou-se pela manobra de "cantilever", a fim de se obter o adequado alinhamento da coluna no plano sagital. Os autores optaram por realizar a hipocorreção da curva torácica nos pacientes Lenke 1C (King 2), a fim

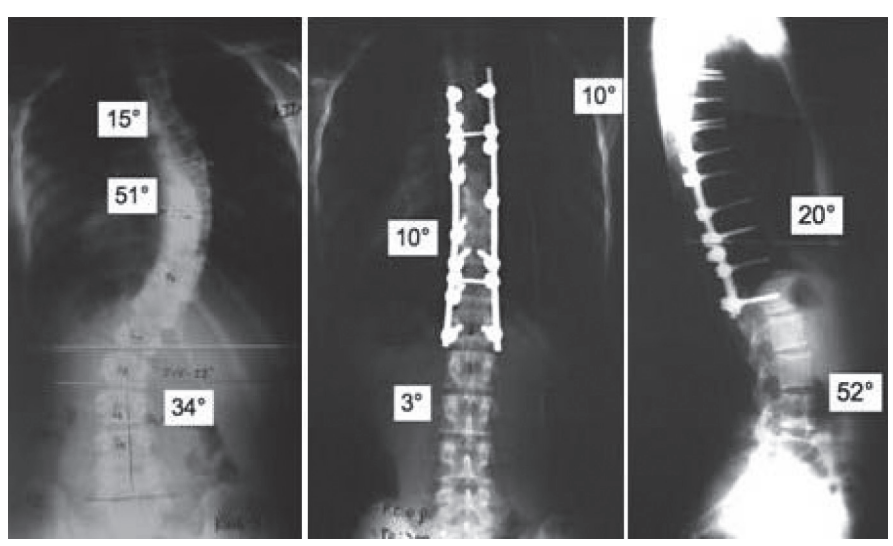

Figura 1. Radiografia em AP pré-operatória e AP+P pós-operatória. 
de evitar a descompensação pós-operatória iatrogênica da curva lombar. Não realizamos monitoração neurofisiológica durante o ato operatório, com potenciais evocados somatossensitivos, por não dispormos de tal equipamento. Realizamos, em todos os casos, o teste do despertar de Stagnara ${ }^{14}$ e não observamos lesão neurológica durante a cirurgia ou no seguimento ambulatorial. Não foram utilizadas órteses após a cirurgia.

Os pacientes foram avaliados através de radiografias panorâmicas pré-operatórias, durante os retornos ambulatoriais e ao final do seguimento. Utilizamos as incidências ântero-posterior (AP) e perfil $(P)$ em posição ortostática e as inclinações laterais direita e esquerda em decúbito dorsal. Todos os casos foram classificados de acordo com King e Moe ${ }^{15}$ e Lenke et al. ${ }^{16}$. Analisamos os parâmetros radiográficos coronais e sagitais, a flexibilidade das curvas, a taxa de correção das deformidades e a inclinação da vértebra inferior instrumentada, vista na radiografia AP no pré e pós-operatório, medida em relação ao plano do solo. Os valores considerados como pós-operatório final datam de maio de 2010, quando todos pacientes foram convocados para reavaliação final. A consolidação da artrodese posterior foi acompanhada através das radiografias realizadas nos retornos ambulatoriais.

A avaliação clínica foi realizada através do questionário Scoliosis Research Society Outcome Instrument (SRS-24) ${ }^{12}$, em versão traduzida para a língua portuguesa, preenchido pelo paciente na avaliação final pós-operatória. Todas as complicações per e pós-cirúrgicas foram descritas durante o acompanhamento dos pacientes.

Os dados foram analisados com o programa estatístico SPSS ${ }^{\circledR}$ versão 13.0 (SPSS Inc.,Chicago, IL, USA). A análise descritiva é mostrada em média, desvio padrão e porcentagens. As variáveis foram testadas quanto a sua normalidade pelo teste Kolmogorov-Smirnov ${ }^{\circledR}$ e apenas o domínio "Satisfação" do SRS-24 não apresentou distribuição normal. Desta forma, para as análises de correlação foram usados os coeficientes de correlação de Pearson ${ }^{\circledR}$ (para as variáveis com distribuição normal) e de Spearman ${ }^{\circledR}$. Foram verificadas as diferenças radiográficas das curvas pré e pós-operatórias por meio do teste $t$ student pareado. Para todas as análises foi usado nível de significância de 0,05.

\section{RESULTADOS}

A amostra foi composta por 25 pacientes que preencheram os critérios de inclusão da pesquisa (Tabela 1).

O tempo de seguimento médio foi de 23,7 meses, variando entre 12 e 35 e a média de idade no momento da cirurgia foi de 14,2 anos (12-17 anos). Do total, 92\% eram do gênero feminino e $8 \%$ masculino.

De acordo com a classificação de King et al. ${ }^{15}, 56 \%$ eram tipo 3 e 20\% tipo 1. Em relação ao lado da curva torácica principal, em 24 casos (96\%) era à direita e apenas em um caso (4\%) à esquerda, sendo que este paciente não pôde ser classificado

Tabela 1. Caracterização da amostra avaliada no estudo.

\begin{tabular}{|c|c|c|c|c|c|c|c|c|c|c|c|}
\hline & \multirow{2}{*}{ Idade } & \multirow{2}{*}{ Risser } & \multicolumn{3}{|c|}{ Cobb Pré-operatório (Inclinação Lateral Pré) } & \multicolumn{3}{|c|}{ Cobb Pós-operatório } & \multicolumn{2}{|c|}{ Classificação } & \multirow{2}{*}{$\begin{array}{l}\text { Tempo } \\
\text { Pós-op. }\end{array}$} \\
\hline & & & Torácica Proximal & Torácica Principal & Lombar & Torácica Proximal & Torácica Principal & Lombar & Lenke & King & \\
\hline 1 & 15 & 4 & $20(10)$ & $56(38)$ & $30(3)$ & 12 & 22 & 5 & IAN & 3 & 35 \\
\hline 3 & 14 & 4 & $58(42)$ & $82(46)$ & $10(0)$ & 38 & 32 & 6 & $2 \mathrm{BN}$ & 5 & 35 \\
\hline 4 & 13 & 4 & $17(2)$ & $50(24)$ & $37(2)$ & 8 & 16 & 22 & $1 \mathrm{CN}$ & 2 & 34 \\
\hline 7 & 12 & 0 & $60(35)$ & $79(40)$ & $33(-14)$ & 44 & 33 & 1 & $2 \mathrm{C}-$ & 5 & 29 \\
\hline 8 & 14 & 3 & $17(8)$ & $48(16)$ & $38(-18)$ & 7 & 9 & 5 & $1 \mathrm{BN}$ & 3 & 26 \\
\hline 9 & 13 & 4 & $15(3)$ & $56(25)$ & $58(30)$ & 9 & 18 & 20 & $6 \mathrm{CN}$ & 1 & 26 \\
\hline 10 & 14 & 4 & $31(7)$ & $60(21)$ & $34(10)$ & 11 & 8 & 14 & $1 \mathrm{BN}$ & 3 & 25 \\
\hline 14 & 16 & 4 & $22(6)$ & $48(27)$ & 41 (15) & 8 & 10 & 14 & $1 \mathrm{CN}$ & NA & 24 \\
\hline 15 & 17 & 4 & $10(5)$ & $34(30)$ & $47(7)$ & 8 & 16 & 9 & $6 \mathrm{CN}$ & 1 & 24 \\
\hline 16 & 13 & 3 & 17 (3) & $39(28)$ & $46(19)$ & 12 & 20 & 26 & $6 \mathrm{CN}$ & 1 & 23 \\
\hline 17 & 15 & 3 & $24(9)$ & 48 (34) & $46(20)$ & 5 & 5 & 11 & $1 \mathrm{CN}$ & 2 & 20 \\
\hline 18 & 15 & 4 & 32 (8) & $64(31)$ & $27(-12)$ & 10 & 15 & 2 & 1AN & 3 & 20 \\
\hline 19 & 14 & 3 & 18 (8) & $46(12)$ & $25(4)$ & 5 & 12 & 1 & $1 \mathrm{AN}$ & 3 & 19 \\
\hline 20 & 17 & 5 & $33(15)$ & 54 (18) & $30(3)$ & 10 & 23 & 20 & $1 \mathrm{BN}$ & 3 & 18 \\
\hline 21 & 16 & 5 & $45(20)$ & $76(36)$ & $46(15)$ & 15 & 23 & 15 & $1 \mathrm{BN}$ & 3 & 14 \\
\hline 22 & 16 & 4 & $25(3)$ & $32(26)$ & 46 (25) & 4 & 5 & 6 & $6 \mathrm{CN}$ & 1 & 12 \\
\hline
\end{tabular}

Fonte: Pacientes do Ambulatório de Afecções da Coluna Vertebral da SCMS. 
segundo King et al. ${ }^{15}$. Segundo a classificação de Lenke et al. ${ }^{16}$, $72 \%$ eram do tipo 1 , sendo que $32 \%$ eram $1 \mathrm{AN}, 24 \% 1 \mathrm{BN}$ e $16 \%$ $1 \mathrm{CN}$. A maioria dos pacientes (48\%) foram classificados como tipo 4, segundo Risser, no momento da cirurgia. Os tipos 3 e tipo 1, representaram $20 \%$ da amostra cada.

A caracterização radiográfica e a porcentagem de correção das curvas dos pacientes são apresentadas na Tabela 2.

Tabela 2. Caracterização das curvas de acordo com a avaliação radiográfica.

\begin{tabular}{|c|c|c|c|c|}
\hline & Média & DP & Mínimo & Máximo \\
\hline \multicolumn{5}{|l|}{ Cobb AP PRÉ } \\
\hline Torácica proximal & 24,4 & 13,5 & 6 & 60 \\
\hline Torácica principal & 54,9 & 12,7 & 32 & 82 \\
\hline Lombar & 38,5 & 10,3 & 10 & 58 \\
\hline \multicolumn{5}{|l|}{ Cobb AP PÓS } \\
\hline Torácica proximal & $11 *$ & 9,8 & 2 & 44 \\
\hline Torácica principal & $16,7^{*}$ & 8,1 & 5 & 33 \\
\hline Lombar & $12,2^{*}$ & 7,8 & 1 & 26 \\
\hline \multicolumn{5}{|l|}{ Cobb Perfil PRÉ } \\
\hline T5-T12 & 27,1 & 9,1 & 9 & 40 \\
\hline L1-L5 & 40,2 & 6,7 & 28 & 58 \\
\hline \multicolumn{5}{|l|}{ Cobb Perfil PÓS } \\
\hline T5-T12 & 27 & 6,2 & 16 & 40 \\
\hline L1-L5 & 41,7 & 4,7 & 34 & 55 \\
\hline \multicolumn{5}{|c|}{$\begin{array}{c}\text { Inclinação da Vértebra } \\
\text { Inferior Instrumentada (IVII) }\end{array}$} \\
\hline Pré & 22,9 & 8,3 & 10 & 40 \\
\hline Pós & $4,9^{*}$ & 4,3 & 0 & 17 \\
\hline \multicolumn{5}{|l|}{ Inclinação Lateral } \\
\hline Torácica proximal & 10,4 & 9,6 & 2 & 42 \\
\hline Torácica principal & 29 & 12,1 & 9 & 60 \\
\hline Lombar & 5,3 & 15,1 & -22 & 33 \\
\hline \multicolumn{5}{|c|}{ Porcentagem de Correção } \\
\hline Torácica proximal & 56,5 & 18,6 & 20 & 88 \\
\hline Torácica principal & 69,9 & 12,2 & 49 & 90 \\
\hline Lombar & 68,7 & 18,8 & 33 & 97 \\
\hline IVII & 79,3 & 15,4 & 43 & 100 \\
\hline
\end{tabular}

${ }^{*}$ Diferenças estatisticamente significativas entre pré e pós-operatório a $p<0,05$.

A média do índice de Cobb na radiografia ântero-posterior pré-operatória foi de $24,4^{\circ}$ para curva torácica proximal, $54,9^{\circ}$ para torácica principal e $38,5^{\circ}$ para curva lombar. A média pós-operatória foi $11,0^{\circ} ; 16,7^{\circ}$ e $12,2^{\circ}$ respectivamente, com porcentagem de correção média da curva torácica principal de 69,9\%. A inclinação da vértebra inferior instrumentada teve correção média de 79,3\% (43 a 100\%). Considerando-se o plano sagital, não observamos diferença significativa entre os valores pré e pós-operatório da cifose medida entre T5 e T12 e da lordose entre L1 e L5. O vaIor médio do questionário SRS foi de 98,1 pontos, variando de 82 a 113 pontos.
Em relação às complicações no pós-operatório, dois pacientes evoluíram com complicações maiores. O paciente número 7 apresentou progressão da deformidade, durante a fase de consolidação da artrodese posterior. Isto foi observado seis meses após o tratamento cirúrgico, com a piora progressiva de aspectos clínicos, como a elevação do ombro esquerdo e a rotação do tronco. Apesar de haver mínima alteração radiográfica, ocorreu piora rotacional da curva torácica proximal, que pôde ser confirmada por estudo tomográfico. Consideramos este o fenômeno do crankshaft, ou virabrequim, uma vez que se tratava de um paciente esqueleticamente imaturo (Risser zero). A conduta foi observacional, não ocorrendo descompensação do tronco ou quebra do material de implante até a estabilização do quadro. Outro caso evoluiu com infecção profunda (paciente 16), que foi operada no $12^{\circ}$ dia pós cirurgia. Realizou-se desbridamento agressivo e coleta de material profundo, identificando a bactéria para antibioticoterapia específica. Obteve-se evolução satisfatória sendo que 17 meses após a cirurgia, devido à recorrência de uma fístula, foi optado pela retirada do material de síntese. No momento intra-operatório observou-se uma artrodese sólida e ausência de infecção profunda.

Não observamos nenhuma soltura ou quebra de parafuso, nem casos de pseudoartrose. Todas as artrodeses posteriores foram consideradas consolidadas em, no máximo, um ano após a cirurgia, com base em parâmetros clínicos e radiográficos. A partir deste momento, todos os pacientes foram liberados para prática de atividades físicas, sem restrições. Nenhuma lesão neurológica (radicular ou medular) foi observada no pós-operatório recente ou tardio.

Correlacionamos os dados da porcentagem de correção e do valor angular pós-operatório da curva (deformidade pósoperatória) torácica principal e lombar com o valor final do questionário SRS-24, além dos domínios satisfação e auto-imagem pós-operatória (Tabela 3).

Não foram encontradas correlações estatisticamente significativas entre nenhuma das variáveis analisadas.

Tabela 3. Análises de correlação entre a avaliação radiográfica e clínica.

\begin{tabular}{c|c|c}
\hline & $\mathbf{R}$ & $\mathbf{p}$ \\
\hline Deformidade Pós-operatória TORÁCICA PRINCIPAL & & \\
\hline SRS 24 total & 0,27 & 0.190 \\
\hline SRS 24 "satisfação" & 0,09 & 0.658 \\
\hline SRS 24 "auto-imagem pós-operatória" & 0,21 & 0.306 \\
\hline Deformidade Pós-operatória LOMBAR & & \\
\hline SRS 24 total & 0,03 & 0.893 \\
\hline SRS 24 "satisfação" & $-0,08$ & 0.703 \\
\hline SRS 24 "auto-imagem pós-operatória" & $-0,32$ & 0.117 \\
\hline \% de Correção TORÁcICA PRINCIPAL & & \\
\hline SRS 24 total & $-0,01$ & 0.936 \\
\hline SRS 24 "satisfação" & 0,03 & 0.905 \\
\hline SRS 24 "auto-imagem pós-operatória" & 0,09 & 0.685 \\
\hline \% de Correção LOMBAR & & \\
\hline SRS 24 total & $-0,03$ & 0.886 \\
\hline SRS 24 "satisfação" & $-0,02$ & 0.917 \\
\hline SRS 24 "auto-imagem pós-operatória" & 0,31 & 0.127 \\
\hline & & \\
\hline & & \\
\hline
\end{tabular}




\section{DISCUSSÃO}

O tratamento da EIA teve importante evolução nas últimas décadas. $\mathrm{O}$ advento do instrumental de terceira geração, descrito por Cotrel e Dubousset ${ }^{5}$ em 1984 e a utilização de PP trouxeram a possibilidade de correção da curva nos três planos espaciais. Há evidências na literatura que comprovam a efetividade e a segurança do uso de PP, inclusive na região torácica. Além aumentar o poder de correção da curva, as montagens apenas com PP levam a menor taxa de soltura do implante e de cirurgias de revisão,

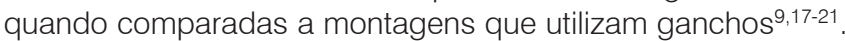

Kim et al. ${ }^{17}$ analisaram 52 pacientes com ElA operados, sendo que em 26 usou-se montagens apenas com PP e nos outros 26 apenas ganchos. A porcentagem de correção da curva torácica principal no grupo em que foram utilizados PP foi de $76 \%$ e no grupo em que se utilizou ganchos foi de 50\%. O mesmo grupo de autores publicou em 2006 outro artigo ${ }^{9}$ que comparou os resultados clínicos e radiográficos de dois grupos de 29 pacientes submetidos ao tratamento cirúrgico de EIA com utilização de montagens híbridas (ganchos e PP) ou somente PP. A porcentagem de correção da curva torácica principal obtida no grupo da montagem híbrida foi de $56 \%$ e no outro grupo de $70 \%$. Apesar da maior correção no grupo em que foram usados apenas parafusos pediculares, não houve diferença estatisticamente significante quanto à avaliação clínica pós-operatória através do questionário SRS-24.

Em nossa amostra observamos uma porcentagem de correção média da curva torácica principal de 69,9\%, semelhante aos dados da literatura supracitados. A correção média da curva torácica proximal foi de $56,5 \%$ e da curva lombar foi de $68,7 \%$. Assim como Lehman et al. ${ }^{22}$ que descreveram a correção da inclinação da vértebra inferior instrumentada (IVII) de 79,4\%, observamos em nossa amostra uma correção de 79,3\%. Entendemos que este parâmetro seja útil para o equilíbrio do tronco no plano coronal, o que poderia elevar os escores de avaliação clínica. A escolha de utilizar somente parafusos monoaxiais (de cabeça fixa) nas montagens foi baseada no maior poder de "derrotação" vertebral que esses parafusos apresentam, quando comparados aos parafusos do tipo poliaxiais, permitindo maior correção da gibosidade ${ }^{21,23}$.

Diab et al. ${ }^{24}$ demonstraram, em uma grande série de casos, o baixo risco de complicações neurológicas com uso de parafusos pediculares. Na análise de 1301 cirurgias para tratamento de EIA encontraram apenas 9 (0,69\%) complicações neurológicas (3 lesões durais, 2 lesões de raiz nervosa e 4 lesões medulares), sendo que todas evoluíram com melhora clínica completa após período máximo de 6 meses. Outros trabalhos também fazem referência à segurança do uso dos parafusos pediculares ${ }^{6-11,13}$. Em nossa amostra não observamos nenhuma lesão neurológica intra ou pós-operatória.
Alguns estudos ${ }^{25-27}$ encontraram relação estatisticamente significativa entre o aumento do escore final do SRS-24 ou de alguns dos seus domínios como auto-imagem e satisfação, com o aumento na porcentagem de correção da curva. A menor deformidade pós-operatória também foi relacionada com o aumento desses escores. Entretanto, não encontramos relação estatisticamente significativa entre a porcentagem de correção da curva torácica principal e lombar, ou seus valores absolutos pós-operatórios, com o escore final do questionário SRS-24. A avaliação individual dos domínios "satisfação" e "auto-imagem pós-operatória" também não apresentou correlação estatisticamente significativa com a porcentagem de correção obtida ou a deformidade final.

D'Andrea et al ${ }^{28}$ também descreveram a ausência de correlação, ou uma correlação fraca entre as variáveis radiográficas e o escore final do SRS-24, em trabalho que avaliou 78 pacientes submetidos ao tratamento cirúrgico da EIA.

Parece lógico pensar que quanto maior a porcentagem de correção da deformidade do paciente, maior será sua satisfação. Entretanto, os dados desse trabalho não nos permitem chegar a essa conclusão. Diversos fatores podem influenciar essa relação. A satisfação do paciente após a cirurgia depende, em grande parte, das suas expectativas pré-operatórias. Fatores como a relação médico-paciente também podem influenciar nesse resultado. Os questionários de avaliação clínica desta pesquisa foram aplicados somente na avaliação final pós-operatória, impossibilitando assim a comparação com dados pré-operatórios. Outras limitações como o pequeno número de casos na amostra e o curto seguimento, a heterogeneidade quanto ao tipo de curva e a ausência de outros parâmetros radiográficos, como compensação do tronco, podem ter influenciado no resultado final da pesquisa. Novos estudos devem ser feitos, englobando avaliações pré e pós-operatórias, com tempo de seguimento maior e incluindo mais variáveis radiográficas para resultados mais confiáveis.

\section{CONCLUSÃO}

Os resultados da correção cirúrgica da EIA utilizando apenas PP mostraram-se estatisticamente significativos em relação à correção angular das curvas e da inclinação da vértebra inferior instrumentada (IVII). A avaliação clínica pós-operatória dos pacientes, feita através do questionário SRS-24, demonstrou um resultado estético-funcional satisfatório. Não foi encontrada relação estatisticamente significante entre a porcentagem de correção da curva (torácica principal ou lombar) ou seu valor angular pós-operatório com o valor final do questionário SRS24 nem com os domínios "satisfação" e "auto-imagem pósoperatória". Observamos baixo índice de complicações, não sendo encontradas complicações neurológicas.

\section{REFERÊNCIAS}

1. Moe JH, Byrd JA. Idiopathic scoliosis. In: Lonsteins JE, Winter RB, Bradford DS RB, Olgivie JW, editors. Moe's textbook of scoliosis and other spinal deformities. 2nd ed. Philadelphia: Saunders; 1987. p.191-232.

2. Petit $\mathrm{Y}$, Aubin $\mathrm{CE}$, Labelle $\mathrm{H}$. Three-dimensional imaging for the surgical treatment of idiopathic scoliosis in adolescents. Can J Surg. 2002;45(6):453-8.

3. Cobb JR. Outline for the study of scoliosis. Instr Course Lect. 1948;5:261-75.

4. Harrington PR. Treatment of scoliosis: correction and internal fixation by spine instrumentation. June 1962. J Bone Joint Surg Am. 2002;84(2):316.

5. Cotrel Y, Dubousset J. Nouvelle technique d'ostéosynthèse rachidienne

segmentaire par voie postérieure. Rev Chir Orthop Reparatrice Appar Mot. 1984;70(6):489-94

6. Suk SI, Lee CK, Kim WJ, Chung YJ, Park YB. Segmental pedicle screw fixation in treatment of thoracic idiopathic scoliosis. Spine (Phila Pa 1976). 1995;20(12): 1399-405.

7. Dobbs MB, Lenke LG, Kim YJ, Kamath G, Peelle MW, Bridwell KH. Selective posterior thoracic fusions for adolescent idiopathic scoliosis: comparison of hooks versus pedicle screws. Spine (Phila Pa 1976). 2006;31(20):2400-4.

8. Takeshita K, Maruyama T, Murakami M, Higashikawa A, Hashimoto H, Hara N, et al. Correction of scoliosis using segmental pedicle screw instrumentation

Coluna/Columna. 2011: 10(2): 91-6 
versus hybrid constructs with hooks and screws. Stud Health Technol Inform. 2006;123:571-6.

9. Kim YJ, Lenke LG, Kim J, Bridwell KH, Cho SK, Cheh G, et al. Comparative analysis of pedicle screw versus hybrid instrumentation in posterior spinal fusion of adolescent idiopathic scoliosis. Spine (Phila Pa 1976). 2006;31(3):291-8.

10. Bullmann V, Liljenqvist UR, Schmidt C, Schulte TL. Posterior operative correction of idiopathic scoliosis. Value of pedicle screws versus hooks. Orthopade. 2009;38(2):198-200, 202-4

11. Liljenqvist UR, Halm HF, LinkTM. Pedicle screw instrumentation of the thoracic spine in idiopathic scoliosis. Spine (Phila Pa 1976). 1997;22(19):2239-45.

12. HaherTR, Gorup JM, Shin TM, Homel P, Merola AA, Grogan DP, et al. Results of the Scoliosis Research Society instrument for evaluation of surgical outcome in adolescent idiopathic scoliosis. A multicenter study of 244 patients. Spine (Phila Pa 1976). 1999;24(14):1435-40.

13. Kim YJ, Lenke LG, Bridwell KH, Cho YS, Riew D. Free hands pedicle screw placement in the thoracic spine: is it safe? Spine (Phila Pa 1976). 2004;29(3):333-42.

14. Vauzelle C, Stagnara P, Jouvinroux P. Functional monitoring of spinal cord activity during spinal surgery. Clin Orthop Relat Res. 1973;(93):173-8.

15. King HA, Moe JH, Bradford DS, Winter RB. The selection of fusion levels in thoracic idiopathic scoliosis. J Bone Joint Surg Am. 1983;65(9):1302-13.

16. Lenke LG, Betz RR, Harms J, Bridwell KH, Clements DH, Lowe TG, et al. Adolescent idiopathic scoliosis: a new classification to determine extent of spinal arthrodesis. J Bone Joint Surg Am. 2001;83(8):1169-81.

17. Kim YJ, Lenke LG, Cho SK, Bridwell KH, Sides B, Blanke K. Comparative analysis of pedicle screw Versus hook instrumentation in posterior spinal fusion of adolescent idiopathic scoliosis. Spine (Phila Pa 1976). 2004;29(18):2040-8.

18. Dobbs MB, Lenke LG, Kim YJ, Kamath G, Peelle MW, Bridwell KH. Selective posterior thoracic fusions for adolescent idiopathic scoliosis: comparison of hooks versus pedicle screws. Spine (Phila Pa 1976). 2006;31(20):2400-4.

19. Kuklo TR, Potter BK, Lenke LG, Polly DW Jr, Sides B, Bridwell KH. Surgical revision rates of hooks versus hybrid versus screws versus combined anteroposterior spinal fusion for adolescentidiopathic scoliosis. Spine (Phila Pa 1976). 2007;32(20):2258-64.

20. Suk SI, Kim WJ, Kim JH, Lee SM. Restoration of thoracic kyphosis in the hypokyphotic spine: a comparison between multiple-hook and segmental pedicle screw fixation in adolescent idiopathic scoliosis. J Spinal Disord.1999;12(6):489-95.

21. Lonner BS, Auerbach JD, Estreicher MB, Kean KE. Thoracic pedicle screw instrumentation. Spine (Phila Pa 1976). 2009;34(20):2158-64.

22. Lehman AR, Lenke LG, Keeler KA, Kim YJ, Buchowski JM, Cheh G, et al. Operative treatment of adolescent idiopathic scoliosis with posterior pedicle screwonly constructs. Spine (Phila Pa 1976). 2008;33(14):1598-604.

23. Kuklo TR, Potter BK, Polly DW, Lenke LG. Monoaxial Versus multiaxial thoracic pedicle screws in the correction of adolescent idiopathic scoliosis. Spine (Phila Pa 1976). 2005;30(18)2113-20.

24. Diab M, Smith AR, Kuklo TR. Neural complications in the surgical treatment of adolescent idiopathic scoliosis. Spine (Phila Pa 1976). 2007;32(24):2759-63.

25. Avanzi O, Landim E, Meves R, Caffaro MFS, Umeta RSG. Escoliose idiopática do adolescente: correlação entre parâmetros radiográficos de correção e resultado clínico de tratamento. Coluna/Columna. 2008;7(3)201-8.

26. Merola AA, Haher TR, Brkaric M, Panagopoulos G, Mathur S, Kohani O, et al. A multicenter study of the outcomes of the surgical treatment of adolescent idiopathic scoliosis using the Scoliosis Research Society (SRS) outcome instrument. Spine (Phila Pa 1976). 2002;27(18): 2046-51.

27. Ali RM, Boachie-Adjei O, Rawlins BA. Functional and radiographic outcomes after surgery for adult scoliosis using third-generation instrumentation techniques. Spine (Phila Pa 1976). 2003;28(11): 1163-9.

28. D'Andrea LP, Betz RR, Lenke LG, Clements DH, Lowe TG, Merola A, et al. Do radiographic parameters correlate with clinical outcomes in adolescent idiopathic scoliosis? Spine (Phila Pa 1976). 2000;25(14):1795-802. 